\title{
Avaliação do princípio da coordenação na atenção primária à saúde da criança em Londrina-PR
}

\section{Evaluation of the principle of coordination in primary health care of the child in Londrina-PR}

\author{
Gisleine Tiemi Souza ${ }^{1}$; Barbara Andrade Alves²; Mauren Teresa Grubisich Mendes \\ Tacla $^{3}$; Neusa Collett ${ }^{4}$; Beatriz Rosana Gonçalves Oliveira Toso ${ }^{5}$
}

\begin{abstract}
Resumo
Atenção Primária à Saúde é um conjunto de funções que fornecem promoção, proteção, prevenção, tratamento e é considerada porta de entrada ao sistema de saúde. Possui quatro atributos essenciais: acesso de primeiro contato, longitudinalidade, integralidade e coordenação. Coordenação é a articulação entre os diversos serviços e ações relacionadas à saúde, que necessitam estar sincronizadas e contínuas para os atendimentos prestados, independentemente do local em que ocorrem. O objetivo do estudo foi avaliar o princípio da coordenação da atenção primária à saúde da criança nas 39 Unidades Básicas de Saúde da área urbana de Londrina-PR. Trata-se de pesquisa multicêntrica, realizada em Cascavel-PR, Londrina-PR e João Pessoa-PB, na forma descritiva e de natureza quantitativa, com a utilização do instrumento PCAToolBrasil, versão criança. Neste instrumento, a coordenação é subdividida em integração de cuidados, que refere à relação entre o serviço básico de saúde e de especialidades; e o sistema de informações que avalia os registros de saúde e a disponibilização dos prontuários. Dos 609 entrevistados, 29,2\% relataram que a criança foi consultada pelo médico especialista. O escore da coordenação - integração de cuidados foi 7,393 e o escore da coordenação - sistema de informações 7,620. Das 8 perguntas do atributo da coordenação que podem ser avaliados por índices numéricos, três perguntas ficaram com escores abaixo de 6,6. Para que o atributo esteja de acordo com os preceitos da atenção primária à saúde, o escore deve estar acima desse valor. Conclui-se que o atributo coordenação obteve alto escore, apesar de três perguntas com escore baixo.
\end{abstract}

Palavras-chave: Atenção primária à saúde. Saúde da criança. Continuidade da assistência ao paciente.

Abstract
Primary Care is a set of universally accessible services that promotes and protects health and prevents and
treats diseases, and it is considered the initial access to the Health System. Four main essential attributes
are present: accessibility, continuity, integrality and coordination. The coordination is the network of
several health related services and actions that must be synchronized and continuous regardless of
the location in which they occur. The goal of this study was to evaluate this coordination principle in

${ }^{1}$ Enfermeira. Residente de Saúde da Criança da Universidade estadual de Londrina. E-mail: gisa_tiemi@hotmail.com

${ }^{2}$ Enfermeira. Mestre em Enfermagem pela Universidade Estadual de Londrina. E-mail: andradealves.b@gmail.com

${ }^{3}$ Enfermeira. Doutora em Enfermagem em Saúde Pública pela Escola de Enfermagem de Ribeirão Preto da Universidade de São Paulo - EERP/USP. Professor Adjunto do Curso de Enfermagem e do Mestrado em Enfermagem da Universidade Estadual de Londrina. E-mail: maurentacla@gmail.com

${ }^{4}$ Enfermeira. Doutora em Enfermagem, docente do Curso de Graduação e do Programa de Pós-Graduação em Enfermagem da Universidade Federal da Paraíba. E-mail: neucollet@gmail.com

${ }^{5}$ Enfermeira. Doutora em Ciências pelo Programa de Enfermagem em Saúde Pública da Escola de Enfermagem de Ribeirão Preto da Universidade de São Paulo - EERP/USP. Professora Adjunta do Colegiado do Curso de Enfermagem da Universidade Estadual do Oeste do Paraná - UNIOESTE, campus de Cascavel. E-mail: lb.toso@certto.com.br 
Primary Care provided to children in 39 UBS (tr. From Basic Health Unit) in the urban area of the city of Londrina. The research is multicentric (Londrina, Cascavel and João Pessoa), descriptive and quantitative - with use of PCATool-Brasil for children. In this instrument, coordination is subdivided in integration of care (which refers to the relation between Basic Health Care and specialties) and the information system (which evaluates the health data and file availability). Amongst the 609 subjects submitted to interview, only $29.2 \%$ reported that a specialist examined the child. The coordinationintegration of care score was 7.393 and the coordination-information system score was 7.620. From the eight questions concerning the coordination attribute that can be numeric-evaluated, three had scores below 6.6. The score should be higher than that to meet the concept of Primary Care. The conclusion is that the coordination attribute had a high score despite the three questions with a low score.

Keywods: Primary health care. Child health. Continuity of patient care.

\section{Introdução}

A Atenção Primária à Saúde (APS) é definida como um conjunto de funções, do nível individual ao coletivo que visa a promoção, prevenção, a entrada do usuário ao sistema de saúde, atendimento direcionado para a pessoa, o diagnóstico precoce, o tratamento de doenças e a reabilitação (HARZHEIM, 2009; STARFIELD, 2002). Segundo Harzheim (2009), a APS ocupa um papel central, que envolveria a organização dos sistemas de saúde para trazer menor custo, mais satisfação por parte do usuário e equidade nos atendimentos.

A APS fundamenta-se em quatro dimensões: acesso de primeiro contato, longitudinalidade, integralidade e coordenação. Sendo a coordenação definida como a articulação dos serviços e ações prestados a pessoa, com direcionamento para a doença, e que a disponibilidade de informações frente aos problemas esteja voltada e sincronizada para um objetivo comum, independentemente do local onde ocorra o atendimento (STARFIELD, 2002).

Como a coordenação possui foco na doença e pode ocorrer em diversos atendimentos e até mesmo, por vários profissionais em diversos níveis de atenção, os serviços de saúde necessitam estar sincronizados através do diálogo contínuo, para solucionar qualquer mal-entendido e evitar ausência de informações para o manejo do caso (STARFIELD, 2002).
Deste modo, a coordenação necessita de um meio para haja as transferências de conhecimentos sobre o paciente e, os métodos para que isso ocorra, podem ser a melhoria da continuidade dos profissionais, atenção compartilhada, prontuários médicos $\mathrm{e}$ informações computadorizadas (STARFIELD, 2002).

Por meio dos meios de transferência de informações, pode-se atingir a continuidade no atendimento, na qual é o elemento estrutural essencial, pois a partir desse elemento o profissional de saúde ficará ciente de todos os problemas apresentados, para um melhor manejo e detecção (STARFIELD, 2002). A continuidade no atendimento é definida por Starfield (2002, p. 390), como "a sucessão de eventos entre as consultas sem importar onde ocorreram e por quais motivos", ou seja, é mais direcionada para o problema, não possui relação pessoal e não é especificada pelo tempo.

Entretanto, para se alcançar a coordenação do cuidado é possível identificar alguns desafios, tais como dentro do estabelecimento da APS (vários membros da equipe com informações diferentes) e com os serviços especializados tanto de curta duração como de longa duração (STARFIELD, 2002). Segundo o Ministério da Saúde (BRASIL, 2007) a coordenação também possui desafios entre os casos de referência e contrarreferência, bem como nos diferentes setores (educação, saneamento, transporte etc.). 
A coordenação pode trazer como vantagens a melhoria da identificação dos problemas de saúde, melhor adesão a tratamentos, consultas de encaminhamento, menos hospitalizações e evitar duplicação desnecessária de exames (STARFIELD, 2002).

A coordenação é de grande relevância para os demais atributos e se esta for aplicada em sua totalidade tem função de coordenar os usuários de saúde para os demais níveis de atendimento, ter a disponibilidade de informação relacionada à pessoa, registros disponíveis e de fácil entendimento para qualquer profissional. Deste modo, o objetivo de estudo foi avaliar o princípio da coordenação da atenção primária à saúde da criança em 39 Unidades Básicas de Saúde da área urbana de Londrina-PR.

\section{Metodologia}

Pesquisa multicêntrica intitulada Avaliação da efetividade da atenção primária em saúde da criança, na qual foram envolvidos os municípios de Londrina e Cascavel, ambos no estado do Paraná, e João Pessoa na Paraíba. Entretanto, este trabalho tem como objetivo apresentar os resultados encontrados no município de Londrina-PR.

Estudo na forma descritiva, natureza quantitativa, realizada no período de agosto de 2012 a janeiro de 2013, com total 609 cuidadores entrevistados nas 39 Unidades Básicas de Saúde (UBS). A população de estudo foi composta por familiares (pai, mãe) e/ou cuidadores (avós, tios, cuidadores legais) de crianças menores de 12 anos.

Foram utilizados dois instrumentos para a entrevista: o sociodemográfico e econômico e o instrumento PCATool-Brasil, versão criança (BRASIL, 2010). O instrumento PCAToolBrasil versão criança apresenta itens do acesso de primeiro contato, longitudinalidade, coordenação, integralidade, orientação familiar e orientação comunitária.

Entretanto, neste trabalho o atributo coordenação que é subdividido em integração de cuidados e sistemas de informações. O atributo coordenação integração de cuidados (item E do instrumento PCATool-Brasil) se refere à relação entre a UBS e o serviço especializado. No total possui 6 questões, porém uma questão é considerada norteadora para continuar essa subdivisão, pois questiona se a criança já consultou com o especialista. Se resposta afirmativa, continuava as perguntas, se não, encerrava essa subdivisão.

As perguntas do atributo coordenação integração de cuidados é composta por: E2 - O (a) "nome do serviço de saúdelou nome médico/ enfermeiro" sugeriu/indicou (encaminhou) que sua criança fosse consultar com este especialista ou serviço especializado?; E3 - O (a) "médico/enfermeiro" da sua criança sabe que ela fez esta consulta com este especialista ou serviço especializado?; E4 - O (a) “médico/ enfermeiro" de sua criança ficou sabendo quais foram os resultados desta consulta?; E5 - Depois desta consulta com o especialista ou serviço especializado, o seu "médico/enfermeiro" conversou com você sobre o que aconteceu durante esta consulta?; E6 - O seu "médico/ enfermeiro" pareceu interessado na qualidade do cuidado que foi dado a sua criança no especialista ou serviço especializado?

Já em relação à subdivisão da coordenação sistema de informações (item $\mathrm{F}$ do instrumento PCATool-Brasil) é frente aos registros de saúde e consulta ao prontuário. Tem no seu total 3 questões, apresentadas a seguir: F1 - Quando você leva sua criança no "nome do serviço de saúdelou nome médico/enfermeiro" você leva algum dos registros de saúde ou boletins de atendimento que a criança recebeu no passado?; F2 - Quando você leva sua criança 
no (a) "nome do serviço de saúdelou nome médico/enfermeiro", o prontuário está sempre disponível na consulta?; F3 - Você poderia ler (consultar) o prontuário/ficha de sua criança se quisesse no "nome do serviço de saúdelou nome médico/enfermeiro".

Foram adotados alguns critérios de inclusão como ser residente da área urbana; ter condições para ser o respondente do formulário, tais como entendimento, expressão e compreensão dos documentos apresentados (TCLE); ser o principal cuidador; ter a idade compatível; ser o cuidador/respondente com conhecimento na unidade que irá avaliar; morar fora da área de abrangência, mas que utiliza o serviço por opção. Por outro lado, foram adotados alguns critérios de exclusão como familiares de crianças de outro município ou que utilizam o serviço de saúde para atividades específicas (imunização).

A pesquisa foi desenvolvida de acordo com as normas da Resolução 196/96 do Conselho Nacional de Saúde, tendo sido aprovada pelo Comitê de Ética em Pesquisa Envolvendo Seres Humanos da Universidade Estadual de Londrina, Parecer CEP/UEL n ${ }^{\circ}$ 061/2012, em 15/05/2012, CAAE $n^{\circ}$ 01295412.2.1001.0107.

\section{Resultados e Discussão}

Participaram da pesquisa 39 UBS das 53 UBS presentes no município de Londrina-PR, totalizando 609 entrevistas respondidas pelos cuidadores/responsáveis das crianças de 0 a menores de 12 anos de idade.

Nos resultados da pesquisa, pode-se observar predominância do filho único em $39,7 \%$ das famílias entrevistadas e a mãe foi a principal cuidadora com 78,8\%. Este resultado foi concordante em estudo realizado por Oliveira (2012), na região metropolitana de Curitiba, em que a mãe era a cuidadora em $84 \%$ nas UBS. Frente a mãe como a principal cuidadora, vale ressaltar o estudo realizado por Santos, Henrique e Silva (2009), que afirma que a mãe é a pessoa que mais sabe cuidar do seu filho e quem, na maioria das vezes, leva a criança à UBS.

Em relação à ocupação, foi encontrado nas famílias participantes $41,4 \%$ dos pais trabalhando formalmente e $35,8 \%$ das mães eram do lar. A renda mensal de dois a quatro salários mínimos $(73,1 \%)$ foi a mais citada. Entretanto, o total de dependentes desta renda mensal, foi de três a cinco pessoas por residência $(85,8 \%)$. Frente os anos de estudo, foi predominante de 10 a 14 anos.

Foi possível identificar predominância da casa própria com $50,0 \%$ e a construção de alvenaria foi a mais citada, com $88,7 \%$. Quanto ao meio de transporte utilizado pela família, o carro foi para 49,9\%, seguido do ônibus coletivo, 42,7\%.

Como já descrito, o atributo coordenaçãointegração de cuidados (E6) possui uma questão norteadora, que envolve se a criança já consultou ou não com o médico especialista. Deste modo, o escore geral deste atributo é quantificado numericamente por meio das outras 5 questões. Segundo Starfield (1992), os escores são definidos a partir da média de cada pergunta, e é considerado alto escore, acima ou igual a 6,6.

Do total dos 609 entrevistados, 29,2\% (178) das crianças passaram pelo médico especialista e, em outro estudo, realizado por Leão, Caldeira e Oliveira (2011), da amostra de 350 crianças menores de 2 anos, 25,2\% relataram consulta com o especialista. Pelo total de consultas com o especialista, porcentagem inferior ao do presente estudo. Já, em outra pesquisa realizada por Almeida, Fausto e Giovanella (2011) evidenciou como positivo os relatos dos médicos e enfermeiros, quanto ao aumento da resolutividade da atenção básica e, que após a implantação da ESF houve queda da procura por serviços hospitalares e especializados. 
Tabela 1 - Escores do atributo coordenação-integração de cuidados (E) realizada nas Unidades Básicas de Saúde da área urbana de Londrina, PR, 2013.

\begin{tabular}{|c|c|}
\hline Perguntas & Escore de $0-10$ \\
\hline $\begin{array}{l}\text { E2 - O (a) "nome do serviço de saúde/ou nome médico/ } \\
\text { enfermeiro" sugeriu/indicou (encaminhou) que sua } \\
\text { criança fosse consultar com este especialista ou serviço } \\
\text { especializado? }\end{array}$ & 8,117 \\
\hline $\begin{array}{l}\text { E3 - O (a) “médico/enfermeiro” da sua criança sabe que } \\
\text { ela fez esta consulta com este especialista ou serviço } \\
\text { especializado? }\end{array}$ & 8,333 \\
\hline $\begin{array}{l}\text { E4 - O "médico/enfermeiro" de sua criança ficou sabendo } \\
\text { quais foram os resultados desta consulta? }\end{array}$ & 7,377 \\
\hline $\begin{array}{l}\text { E5 - Depois desta consulta com o especialista ou serviço } \\
\text { especializado, o seu “médico/enfermeiro" conversou com } \\
\text { você sobre o que aconteceu durante esta consulta? }\end{array}$ & 6,553 \\
\hline $\begin{array}{l}\text { E6 - O seu "médico/enfermeiro" pareceu interessado } \\
\text { na qualidade do cuidado que foi dado a sua criança no } \\
\text { especialista ou serviço especializado? }\end{array}$ & 6,573 \\
\hline Escore do componente & 7,393 \\
\hline
\end{tabular}

Fonte: Autores.

O escore que a coordenação-integração de cuidados atingiu foi 7,393 (Tabela 1), sendo considerado alto escore (STARFIELD, 1992). Vale ressaltar o estudo realizado por Oliveira (2012), onde a ESF atingiu escore semelhante a essa pesquisa. Porém, o estudo de Oliveira (2012) dividiu os escores em UBS e ESF, mas neste estudo não foi possível separar os escores, pois, as unidades básicas estudadas estão dispostas da ESF, sendo assim, analisaram-se os dados com a ESF.

Como se observa na tabela 1 , duas perguntas ficaram com baixo escore, sendo elas: após a consulta com o especialista o médico/enfermeiro conversou sobre o que aconteceu durante esta consulta, atingindo escore 6,553, e o médico/ enfermeiro pareceu interessado na qualidade do cuidado que foi dado a criança no especialista, atingindo escore 6,573.

Corroborando com este estudo, o estudo realizado por Oliveira (2012) atingiu escore de 6,316 na ESF na pergunta referente se o profissional de saúde conversou sobre o que aconteceu durante a consulta. Evidencia escores inferiores ao linear em ambos os estudos.

Por outro lado, a pergunta frente ao interesse na qualidade do cuidado que foi oferecido no especialista, em comparação com o estudo de Oliveira (2012) que obteve escore de 7,719 na ESF, este estudo apresentou escore inferior ao linear. Entretanto, quando questionados sobre se o médico da atenção básica sabia sobre a consulta com o especialista, o escore foi de 8,333.

Pode-se inferir, que os baixos escores encontrados no estudo vão ao oposto dos preceitos da APS, já que a atenção primária possui foco na família e as ações devem ser atreladas conforme os princípios da humanização do cuidado, mas após o surgimento da Política Nacional de Humanização da atenção, que tem como um dos propósitos o desenvolvimento de relações e compartilhamento das práticas de gestão e atenção, fortalecimento da humanização e a implantação de métodos de acompanhamento e avaliação (BRASIL, 2014; COELHO; JORGE, 2009).

Conforme o que se apresenta na Tabela 1 as demais perguntas que podem ser quantificadas 
numericamente, apresentaram escore acima do linear, sendo concomitante ao estudo realizado por Oliveira (2012), que encontrou resultados similares a essa pesquisa. Já, na Tabela 2, a coordenaçãosistema de informações apresenta três questões e as três, são possíveis quantificações numéricas, sendo que o escore deste atributo atingiu 7,620, sendo considerado alto escore (STARFIELD, 1992). Em comparação com o estudo de Oliveira (2012), que atingiu escore 6,30 nas ESF e 5,16 nas UBS, indicou a superioridade de Londrina nesse atributo.

Obteve apenas uma questão com baixo escore, na qual a pergunta se referia à consulta/leitura do prontuário da criança, atingindo escore 4,700. Devido ao baixo escore, pode-se entender que muitas vezes o usuário e/ou o responsável não sabe que possui acesso livre aos dados do prontuário médico, e que possui respaldo, tanto do inciso VIII do artigo $2^{\circ}$ que reafirma a constituição, como no Código de Ética Médica (OSELKA, 2001).

Quando perguntado sobre a disponibilidade dos prontuários, o escore atingiu 9,223, sendo este, o escore mais alto desta subdivisão (Tabela 2). Este estudo apresentou superioridade ao ser comparado com Oliveira (2012), pois tanto na $\operatorname{ESF}(5,840)$ quanto na UBS $(5,185)$, o escore foi abaixo do linear.

Devido ao alto escore da disponibilidade dos prontuários na APS, os prontuários podem apoiar a melhoria da continuidade do cuidado, e como já foi citado, o prontuário é considerado um meio para transferência de informação do paciente, mas que para ser efetivo, necessita de informações pertinentes e importantes sobre o paciente (STARFIELD, 2002).

Tabela 2 - Escores do atributo coordenação - Sistema de Informações (F) realizado nas Unidades Básicas de Saúde na área urbana de Londrina, PR, 2013.

\begin{tabular}{lc}
\hline \multicolumn{1}{c}{ Perguntas } & Escore de 0-10 \\
\hline $\begin{array}{l}\text { F1 - Quando você leva sua criança no “nome do } \\
\text { serviço de saúde/ou nome médico/enfermeiro” }\end{array}$ & \\
você leva algum dos registros de saúde ou boletins & 8,940 \\
de atendimento que a criança recebeu no passado? & \\
F2 - Quando você leva sua criança no (a) "nome & \\
do serviço de saúde/ou nome médico/enfermeiro”, & 9,223 \\
o prontuário dela está sempre disponível na & \\
consulta? & \\
F3 - Você poderia ler (consultar) o prontuário/ & \\
ficha de sua criança se quisesse no “nome do & \\
serviço de saúdelou nome médico/enfermeiro”? & \\
Escore do componente & 7,620 \\
\hline
\end{tabular}

Fonte: Autores.

\section{Conclusão}

Tendo em vista o objetivo de estudo e a importância do atributo coordenação na APS, foi possível observar altos escores em ambas às subdivisões, ou seja, segundo a percepção dos usuários entrevistados o atributo é capaz de exercer sua função, que é coordenar o sistema de saúde, principalmente em relação à consulta com o especialista.

No entanto, segundo visão dos usuários entrevistados, algumas perguntas apresentaram escore inferior ao linear, o que permite evidenciar a fragilidade de alguns itens na atenção primária à saúde 
da criança, principalmente quando relacionado ao cuidado integral prestado no serviço especializado.

Nota-se baixa referência para o serviço especializado, que tanto pode estar relacionado com o aumento da resolutividade das unidades básicas de saúde, como pode estar relacionado à idade da criança. Em relação à idade da criança, não foi quantificado, sendo considerada uma fragilidade para concluir a baixa referência e a fragilidade do estudo.

O estudo nos mostrou alta disponibilidade dos prontuários de saúde nas UBS, e visto que é considerado um meio para comunicação entre os profissionais de saúde, seria adequada a melhoria das informações descritas no mesmo, pois muitas informações ficam restritas à pessoa que escreveu, dificultando a continuidade do atendimento ao paciente.

Mesmo o atributo coordenação com alto escore, segundo os usuários entrevistados, conclui-se que é necessária a reflexão por parte dos trabalhadores da saúde na atenção primária para as perguntas de baixo escore e também, para os gestores, a fim de motivar e educar os profissionais para assim, garantir a totalidade na assistência ao cuidado da saúde infantil.

\section{Agradecimento}

Agradeço a Universidade Estadual de Londrina pela disponibilização da bolsa de Iniciação Científica.

\section{Referências}

ALMEIDA, P. F.; FAUSTO, M. C. R.; GIOVANELLA, L. Fortalecimento da atenção primária à saúde: estratégia para potencializar a coordenação dos cuidados. Revista Panamericana Salud Publica, Washington, v. 29, n. 2, p. 84-95, 2011.

BRASIL. Conselho Nacional de Secretários de Saúde. Atenção primária e promoção da saúde. Conselho Nacional de Secretários de Saúde. Brasília: CONASS, 2007.
- Ministério da Saúde. Humaniza SUS: política nacional de humanização. 2014. Disponível em: <http://portalsaude.saude.gov.br/index.php/oministerio/principal/secretarias/sas/humanizasus $>$. Acesso em: 27 nov. 2014.

Ministério da Saúde. Secretaria de

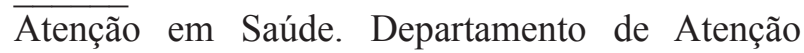
Básica. Manual do instrumento de avaliação da atenção primária à saúde: primary care assessment tool pcatool-Brasil. Brasília: Ministério da Saúde, 2010. 80 p.

COELHO, M. O.; JORGE, M. S. B. Tecnologia das relações como dispositivo do atendimento humanizado na atenção básica à saúde na perspectiva do acesso, do acolhimento e do vínculo. Ciência \& Saúde Coletiva, Rio de Janeiro, v. 14, supl 1, p. 1523-1531, 2009.

HARZHEIM, E. Texto referência: atenção primária na saúde (APS). In: CICLO DE DEBATES SOBRE REDES REGIONALIZADAS DE ATENÇÃO À SAÚDE: DESAFIOS DO SUS, 2009, Brasília. Anais... Brasília: OPAS, 2009.

LEÃO, C. D.A.; CALDEIRA, A. P.; OLIVEIRA, M. M. C. Atributos da atenção primária na assistência à saúde da criança: avaliação dos cuidadores. Revista Brasileira Materno-Infantili, Recife, v. 11, n. 3, p. 323-334, jul./set. 2011.

OLIVEIRA, V. B. C. A. Avaliação da atenção primária à saúde da criança no município de Colombo-PR. 2012. 123 f. Dissertação (Mestrado) Escola de Enfermagem, Universidade de São Paulo, São Paulo, 2012.

OSELKA, G. Direitos dos pacientes e legislação. Revista da Associação Médica Brasileira, São Paulo, v. 47, n. 2, p. 104-105, 2001.

SANTOS, M. A.; HENRIQUE, V. C.; SILVA, V. C. A compreensão das mães a cerca da consulta de puericultura numa unidade saúde da família. Rede de Cuidados em Saúde, Rio de Janeiro, v. 3, n. 3, p. 1-14, 2009.

STARFIELD, B. Atenção primária: equilíbrio entre necessidades de saúde, serviços e tecnologia. Brasília: UNESCO, 2002. 
Primary care: concept, evaluation and

policy. New York: Oxford University Press, 1992. 